\title{
Langmuir
}

pubs.acs.org/Langmuir

(C) 2009 American Chemical Society

\section{Ion Specific Electrolyte Effects on Thin Film Drainage in Nonaqueous Solvents Propylene Carbonate and Formamide}

\author{
Christine L. Henry, ${ }^{\dagger}$ Stoyan I. Karakashev, ${ }^{\star}$ Phong T. Nguyen, ${ }^{\star}$ Anh V. Nguyen, ${ }^{\star}$ and \\ Vincent S. J. Craig*, \\ ${ }^{\dagger}$ Department of Applied Mathematics, Research School of Physics and Engineering, \\ The Australian National University, Canberra ACT 0200, Australia, and Division of Chemical Engineering, \\ The University of Queensland, Brisbane, Queensland 4072, Australia
}

Received March 29, 2009. Revised Manuscript Received May 18, 2009

\begin{abstract}
Electrolytes have been found to stabilize thin films in nonaqueous solvents propylene carbonate and formamide, in the absence of surfactant. The thin film balance microinterferometry technique has been used to measure film lifetimes, drainage kinetics, and rupture thicknesses for thin films between air-nonaqueous solution interfaces. Electrolytes that were previously found to inhibit bubble coalescence in bulk bubble column measurements also increase the lifetimes of individual thin films across a similar concentration range (from 0 to $0.3 \mathrm{M}$ ). We report that increasing the concentration of inhibiting electrolyte stabilizes the thin liquid film in two ways: the rate of film drainage decreases, and the film reaches a lower thickness before rupturing. In contrast, noninhibiting electrolyte shows little to no effect on film stability. We have here demonstrated that both drainage and rupture processes are affected by the addition of electrolyte and the effect on the thin film is thus ion specific.
\end{abstract}

\section{Introduction}

It is well-known that simple electrolytes, at sufficiently high concentrations, can stabilize bubbles against coalescence in aqueous solution. ${ }^{1-4}$ This phenomenon is seen, for example, in the froth that forms in seawater but not in freshwater, ${ }^{5}$ however, despite this ease of observation, the mechanism behind electrolyte stabilization of bubbles remains unknown. ${ }^{6}$ The inhibition is also ion specific, with some salts inhibiting coalescence at around $0.1 \mathrm{M}$, while others show no effect up to $0.5 \mathrm{M}$. It has been shown that bubble coalescence inhibition depends upon the combination of cation and anion present. ${ }^{1,2}$ Bubble coalescence inhibition was codified by empirically assigning the ions a property $\alpha$ or $\beta$. An electrolyte would then be represented by the property of the cation and anion. It was shown that $\alpha \alpha$ and $\beta \beta$ electrolytes inhibit coalescence; however, the $\alpha \beta$ and $\beta \alpha$ salts had no effect. The origin of these combining rules and the properties or behavior of an ion that relate to the $\alpha$ and $\beta$ categories are still unclear. It is believed that the specific ion effects exhibited in bubble coalescence should be related to the numerous specific ion effects known as Hofmeister effects, ${ }^{6}$ but this is yet to be elucidated.

Recently, some of us extended the applicability of ion combining rules, when we reported the inhibition of bubble coalescence in nonaqueous solvents, at similar concentrations to those reported in aqueous solution. ${ }^{7}$ In particular, the high-dielectric solvents propylene carbonate and formamide each showed evidence of electrolyte inhibition of bubble coalescence that was ion

*To whom correspondence should be addressed. E-mail: vince.craig@anu. edu.au.

(1) Craig, V. S. J.; Ninham, B. W.; Pashley, R. M. J. Phys. Chem. 1993, 97, 10192-10197.

(2) Craig, V. S. J.; Ninham, B. W.; Pashley, R. M. Nature 1993, 364, 317-319.

(3) Foulk, C. W.; Miller, J. N. Ind. Eng. Chem. 1931, 23, 1283.

(4) Lessard, R. R.; Zieminski, S. A. Ind. Eng. Chem. Fundam. 1971, 10(2), 260269.

(5) Winkel, E. S.; Ceccio, S. L.; Dowling, D. R.; Perlin, M. Exp. Fluids 2004, 37, 802-810.

(6) Craig, V. S. J. Curr. Opin. Colloid Interface Sci. 2004, 9, 178-184.

(7) Henry, C. L.; Craig, V. S. J. Langmuir 2008, 24, 7979-7985. specific and followed combining rules. In formamide, the $\alpha$ and $\beta$ assignments match those in water, while in propylene carbonate the assignments differ. That work was carried out in a bubble column, and the change in bubble coalescence was therefore observed as the average of many individual collisions. An alternative approach is to study the stability of a single thin film between gas interfaces, which can reveal more of the mechanism by which bubble coalescence is inhibited. ${ }^{8}$ Coalescence during the lifetime of a bubble collision involves both drainage of the thin liquid film between gas surfaces, and rupture of the thin film. Coalescence inhibition may act on either or both of these processes.

In this article, we report measurements on thin films of the nonaqueous solvents propylene carbonate and formamide in the presence of electrolytes. The drainage and rupture of the thin films of liquid in air are studied using microinterferometry. We have chosen electrolytes that are found to dramatically reduce bubble coalescence at concentrations below $\sim 0.1 \mathrm{M}$ and electrolytes that have little or no effect on bubble coalescence at these concentrations; these are described as inhibiting and noninhibiting electrolytes, respectively. ${ }^{7}$ We aimed to elucidate, first, how inhibiting electrolyte affects thin film lifetime, drainage, and rupture thickness and, second, how thin films differ in inhibiting and noninhibiting electrolyte solution. We also wished to investigate how the drainage observed in electrolyte solutions compares to theories of drainage kinetics that were developed to explain surfactant systems.

\section{Experimental Section}

Materials and Methods. Thin films in nonaqueous solvents in the presence of electrolyte were studied using a microinterferometric setup. This apparatus is shown in Figure 1. The film was formed in a Scheludko cell ${ }^{9}$ connected via capillary to a gastight

(8) Karakashev, S. I.; Nguyen, P. T.; Tsekov, R.; Hampton, M. A.; Nguyen, A. V. Langmuir 2008, 24, 11587-11591.

(9) Sheludko, A. Adv. Colloid Interface Sci. 1967, 1, 391-464 


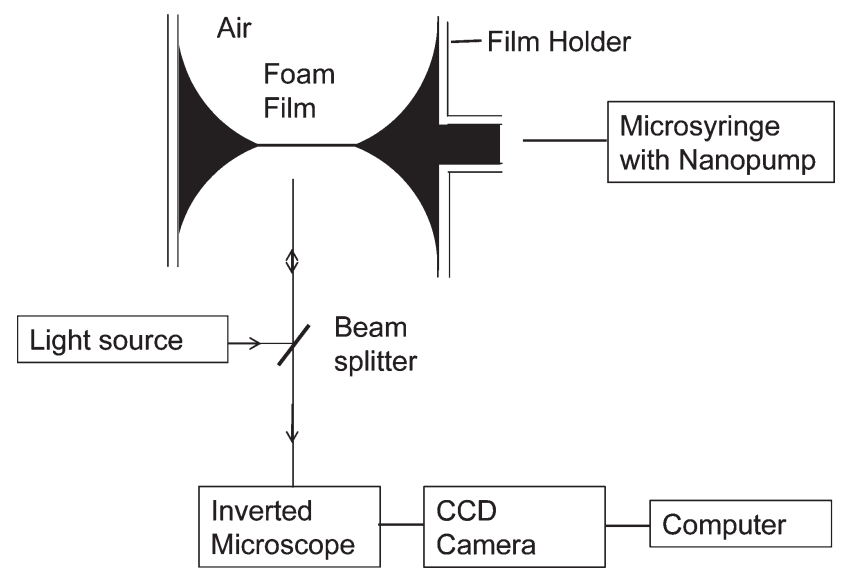

Figure 1. Thin film balance apparatus. A planar film (not to scale) is formed in the Scheludko cell film holder ${ }^{9}$ (4 mm internal diameter) by withdrawing liquid using a microsyringe. The interferometric data is obtained using an inverted microscope and recorded to a computer using a CCD camera.

microsyringe pump. An inverted microscope (Nikon, Japan) was used for illuminating and observing the film and reflected interference fringes. A CCD video camera recorded images at 30 frames per second. The images were stored to a computer and later analyzed.

The thin film was created by injecting the liquid of interest into the film holder (diameter $4 \mathrm{~mm}$ ) and then withdrawing the liquid using a microsyringe pump. When the planar film was formed (interference fringes were observed), the pumping was stopped and the film was left to drain until rupture. At least 20 film drainage events were recorded in each solution. In between each drainage and rupture event, liquid was pumped back into the film holder to form a stable double-concave drop, from which liquid was then withdrawn to form a new film. The withdrawal rate was varied (between $20 \mathrm{~nL} / \mathrm{s}$ and $1000 \mathrm{~nL} / \mathrm{s}$ ) to obtain films of different radius. Film lifetime data (the time from the formation of a planar film with visible interference fringes, until film rupture) were collected for films with $20 \mu \mathrm{m} \leq R \leq 300 \mu \mathrm{m}$. In some cases, film thickness was measured so that drainage kinetics could be determined; the analysis required for determination of film thickness and drainage kinetics is presented below. During an experiment, the solution in the film holder was replaced at least once, in order to minimize possible contamination of the surface. The cell was left sealed for at least 5 min to allow the cell atmosphere to equilibrate after each fluid replacement. The interval between the time when fresh solution was collected and the cell was closed, and the time at which a film measurement was taken, was recorded. This interval is defined as the droplet age because it is a measure of the time for which that solution droplet has been present in the cell holder of the thin film balance. As discussed below, we observed a dependence of film lifetime (film stability) on droplet age in the case of some of the formamide solutions.

Thin films were measured in inhibiting and noninhibiting electrolytes, as found in bubble coalescence measurements. ${ }^{7}$ The molecular structures of the solvents, and relevant physical properties, are given in the Supporting Information. In propylene carbonate, inhibiting salts $\mathrm{LiBr}$ and $\mathrm{NaSCN}$ and noninhibiting electrolyte $\mathrm{HCl}$ (as the $35 \% \mathrm{w} / \mathrm{w}$ aqueous solution) were used. In formamide, solutions of inhibiting electrolyte $\mathrm{LiCl}$ and noninhibiting electrolyte $\mathrm{CH}_{3} \mathrm{COONa}$ were used. Sodium acetate was dried at $250{ }^{\circ} \mathrm{C}$ for $5 \mathrm{~h}$ to remove water; other salts were used as received. Electrolyte concentrations covered the range of concentrations used in bubble column measurements $(0.01-$ $0.3 \mathrm{M}$ ); solutions were diluted from a concentrated stock solution. For stock solutions of $\mathrm{CH}_{3} \mathrm{COONa}$ and $\mathrm{LiCl}$ in formamide, an additional cleaning step was employed. The solutions underwent sparging for $1 \mathrm{~h}$ with $\mathrm{N}_{2}$ gas, following a method that was outlined by Brandon et al. ${ }^{10}$ and recently employed to purify solutions for bubble rise experiments. ${ }^{11}$ The sparging bubbles cleaned the solution by collecting any surface active contaminant and depositing it on the surface of the liquid and on the glass sides of the sparging vessel, which became noticeably nonwetting during the cleaning process. The fluid was removed from the bulk with a syringe, without passing through the liquid interface, where surface active contaminants were concentrated. The prepared solutions were kept at room temperature $\left(\sim 20^{\circ} \mathrm{C}\right)$. The $\mathrm{NaSCN}$ solutions were kept in the dark because the thiocyanate is light-sensitive.

The thin films contain a small solution volume and have surfaces that are exposed to air for some time. The system is thus extremely susceptible to low levels of surfactant contamination, and cleaning of the Scheludko cell is important. The cell (film holder and capillary) was rinsed many times using a mixture of ethanol/water/KOH $(84: 16: 12.5)$. Sometimes RCA solution (made up as $50 \mathrm{~mL}$ of $\mathrm{H}_{2} \mathrm{O} / 10 \mathrm{~mL}$ of $28 \% \mathrm{NH}_{3} / 10 \mathrm{~mL}$ of $30 \%$ $\left.\mathrm{H}_{2} \mathrm{O}_{2}\right)$ at $\geq 70^{\circ} \mathrm{C}$ was employed. After rinsing, the cleanness of the cell was checked in pure water. In a system free of organic contaminant, the film is very unstable and no interference fringes are observed. Water was removed from the cell by rinsing with absolute ethanol and drying with $\mathrm{N}_{2}$ gas. The neat solvent (formamide or propylene carbonate) was then used to rinse the film holder and capillary. A small amount of solvent was put in the base of the cell, to create a saturated atmosphere when the cell was sealed.

Film Thickness Determination. The film thickness can be determined via microinterferometry. For those cases in which $R \leq 40 \mu \mathrm{m}$, the thin film is of uniform thickness within the meniscus, and can be treated using drainage models developed for plane parallel interfaces. It is then possible to compare experimental drainage kinetics and rupture thicknesses with theoretical predictions. Larger films show regions of deformation and nonuniform thickness ${ }^{12}$ and are not suitable for this analysis. The film is illuminated with white coherent light $(150 \mathrm{~W})$. This light is reflected off both film surfaces, creating an interference pattern (Newton rings) with color being dependent upon the film thickness. To determine the film thickness, the interferometric images were processed by a digital filtration procedure using software for digital processing (Optimas 6.1, Optimas) and a digital green filter with wavelength $\lambda=546 \mathrm{~nm} .{ }^{13}$ This procedure converted the polychromatic into monochromatic interferograms suitable for calculating the film thickness. The film thickness, $h$, is calculated using the interferometric equation: ${ }^{9,14}$

$$
h=\frac{\lambda}{2 \pi n}\left[l \pi \pm \arcsin \sqrt{\frac{\Delta\left(1+r^{2}\right)}{\left(1-r^{2}\right)+4 r^{2} \Delta}}\right]
$$

In eq 1 , the Fresnel reflection coefficient, $r=(n-1)^{2} /(n+1)^{2}$, for the air-solution interface is a function of the refractive index, $n$, of the film solution; $l=0,1,2, \ldots$, is the order of interference; and $\Delta=\left(I-I_{\min }\right) /\left(I_{\max }-I_{\min }\right)$, where $I$ is the instantaneous intensity of the photocurrent and $I_{\min }$ and $I_{\max }$ are its minimum and maximum values, respectively. The refractive index used was that for the neat solvents (see the Supporting Information), as it was assumed that any refractive index change due to added electrolyte at $\leq 0.3 \mathrm{M}$ will be small and have minimal effect on the film thickness calculation.

(10) Brandon, N. P.; Kelsall, G. H.; Levine, S.; Smith, A. L. J. Appl. Electrochem. 1985, 15, 485-493.

(11) Henry, C. L.; Parkinson, L.; Ralston, J. R.; Craig, V. S. J. J. Phys. Chem. C 2008, 112, 15094-15097.

(12) Manev, E. D.; Nguyen, A. V. Adv. Colloid Interface Sci. 2005, 114-115, 133-146.

(13) Karakashev, S. I.; Nguyen, A. V.; Manev, E. D. J. Colloid Interface Sci. 2007, 306, 449-453.

(14) Nguyen, A. V.; Schulze, H. J. Colloidal Science of Flotation, 1st ed.; Marcel Dekker: New York, 2004; p 840. 


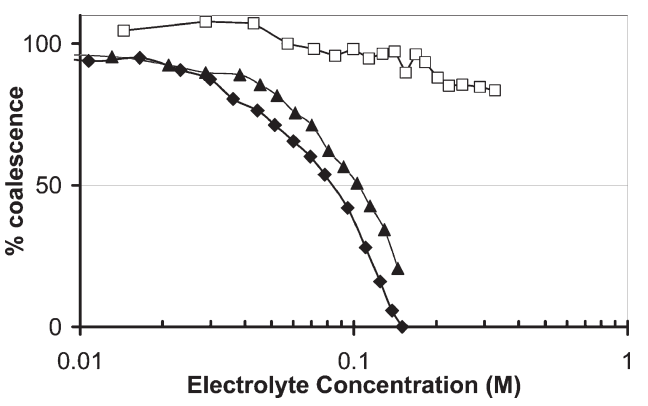

Figure 2. Bubble column results for electrolytes used in propylene carbonate thin film drainage: inhibiting electrolytes $\operatorname{NaSCN}(\bullet)$ and $\mathrm{LiBr}(\mathbf{\Delta})$, and noninhibiting electrolyte $\mathrm{HCl}(\square) .100 \%$ coalescence is defined as in pure solvent; $0 \%$ coalescence is a stable, low voltage signal in inhibiting electrolytes. These results have been previously reported.

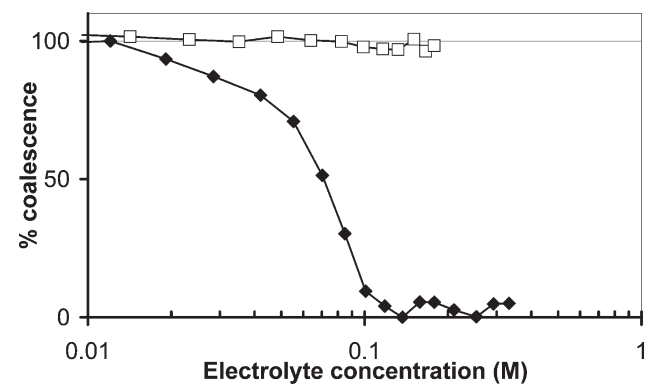

Figure 3. Bubble column results for electrolytes used in formamide thin film drainage: inhibiting electrolyte $\mathrm{LiCl}(\bullet)$ and noninhibiting electrolyte $\mathrm{CH}_{3} \mathrm{COONa}(\square) .100 \%$ coalescence is defined as in pure solvent; $0 \%$ coalescence is a stable, low voltage signal in inhibiting electrolytes. These results have been previously reported. ${ }^{7}$

\section{Results and Discussion}

The effects of the various electrolytes on bubble coalescence in a bubble column are shown in Figures 2 and 3 for solvents propylene carbonate and formamide, respectively. The coalescence is measured as bulk turbidity in the column, based on scattering of an expanded, collimated laser beam. Inhibition of coalescence by electrolytes leads to more, smaller bubbles, greater scattering, and lower transmitted laser intensity. The apparatus has been previously described. ${ }^{1,15}$ A percentage scale is used to compare electrolyte effects. $100 \%$ coalescence is defined in pure solvent, while $0 \%$ coalescence is a stable, low value reached in inhibiting electrolytes.

Thin films were studied in electrolyte concentrations from $0 \mathrm{M}$ to either 0.2 or $0.3 \mathrm{M}$, as this is the range over which inhibiting electrolytes change the bubble stability. We hoped to gain more information about how thin film lifetime changes as a function of electrolyte concentration. Bubble coalescence inhibition in the bubble column occurs when the duration of a bubble collision is less than the time required for film drainage and rupture processes. It is hypothesized that an increase of average film lifetime will be observed with increasing inhibiting electrolyte concentration, in single thin films. By analyzing drainage kinetics and film rupture thickness of single thin films, we hoped to learn more of the mechanism by which electrolytes inhibit bubble coalescence.

Thin Film Lifetimes in Electrolyte Solutions. The effects on propylene carbonate film lifetime of inhibiting electrolytes $\mathrm{NaSCN}$ and $\mathrm{LiBr}$, and noninhibiting electrolyte $\mathrm{HCl}$ (added as

(15) Henry, C. L.; Dalton, C. N.; Scruton, L.; Craig, V. S. J. J. Phys. Chem. C 2007, 111, 1015-1023.

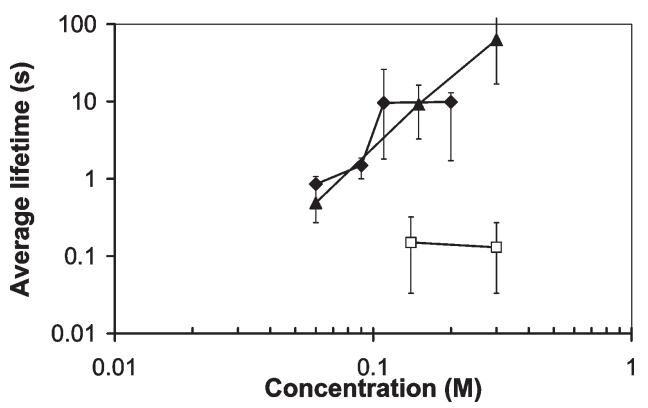

Figure 4. Average lifetime of thin films as a function of concentration in inhibiting electrolytes $\mathrm{NaSCN}(\bullet)$ and $\operatorname{LiBr}(\boldsymbol{\Delta})$, and noninhibiting electrolyte $\mathrm{HCl}(\square)$ in propylene carbonate plotted on a $\log -\log$ plot. Lifetime increases with increasing inhibiting salt. Lower concentrations showed no visible fringes and hence have a film lifetime of $0 \mathrm{~s}$. Lifetime error bars show the range between 10th and 90th percentiles at each concentration, for $21 \leq N \leq 47$. Film radius is varied at each concentration.

concentrated aqueous solution) were measured at several concentrations. In pure propylene carbonate, no stable film was observed, as predicted for pure liquids, ${ }^{16}$ and in most cases no interference fringes could be seen before the film ruptured. This indicates rupture occurred rapidly before the film had drained below $200 \mathrm{~nm}$. The average lifetimes as a function of electrolyte concentration for the three electrolytes are shown in Figure 4. Increasing the concentration of inhibiting electrolyte led to longer-lived films, whereas increasing the concentration of $\mathrm{HCl}$ (a noninhibiting electrolyte) up to $0.3 \mathrm{M}$ had little effect. The concentration range over which an increase in film lifetime is seen corresponds well with the bubble coalescence inhibition results shown in Figure 2.

Histograms showing film lifetime variability for two intermediate concentrations of inhibiting salt NaSCN in propylene carbonate are shown in Figure 5. At $0.06 \mathrm{M} \mathrm{NaSCN}$, thin films were stabilized relative to pure solvent but rapidly drained and ruptured with an average lifetime of $0.86 \mathrm{~s}$ for this sample $(N=$ $25)$. Coalescence is more inhibited at $0.11 \mathrm{M} \mathrm{NaSCN}$, and this is consistent with increase in film average lifetime to $9.6 \mathrm{~s}(N=25)$ as well as the existence of some long-lived films of over $60 \mathrm{~s}$. However, the increased average film lifetime at higher concentrations is not simply a product of a few very long-lasting films: even the minimum lifetime of small films was shown to increase with increasing inhibiting electrolyte.

It will be observed in Figure 4 that there is a difference in lifetime at the higher concentrations of inhibiting electrolyte, with $\mathrm{LiBr}$ continuing to increase in film lifetime while NaSCN shows no significant change on increasing concentration from 0.11 to $0.20 \mathrm{M}$ solution. The behavior in this high concentration regime is not available for comparison in the bubble column experiments, because once the conditions are such that film lifetime is longer than bubble collision lifetime, coalescence is inhibited and little further variation can be seen. It would certainly be of interest to ascertain whether higher concentrations lead to increasingly stable films or whether a maximum lifetime is reached. In aqueous solutions in a closed cell, Karakashev et al. have reported that films even at $4 \mathrm{M} \mathrm{NaCl}$ rupture with a lifetime of tens of seconds, suggesting that lifetime does not continue to increase. ${ }^{8}$

Thin film lifetimes were measured in inhibiting electrolyte $\mathrm{LiCl}$ and noninhibiting electrolyte $\mathrm{CH}_{3} \mathrm{COONa}$ in formamide solutions. The average film lifetimes are shown in Figure 6. The average lifetime in lithium chloride is seen to be higher at all

(16) Li, D.; Liu, S. Langmuir 1996, 12, 5216-5220. 

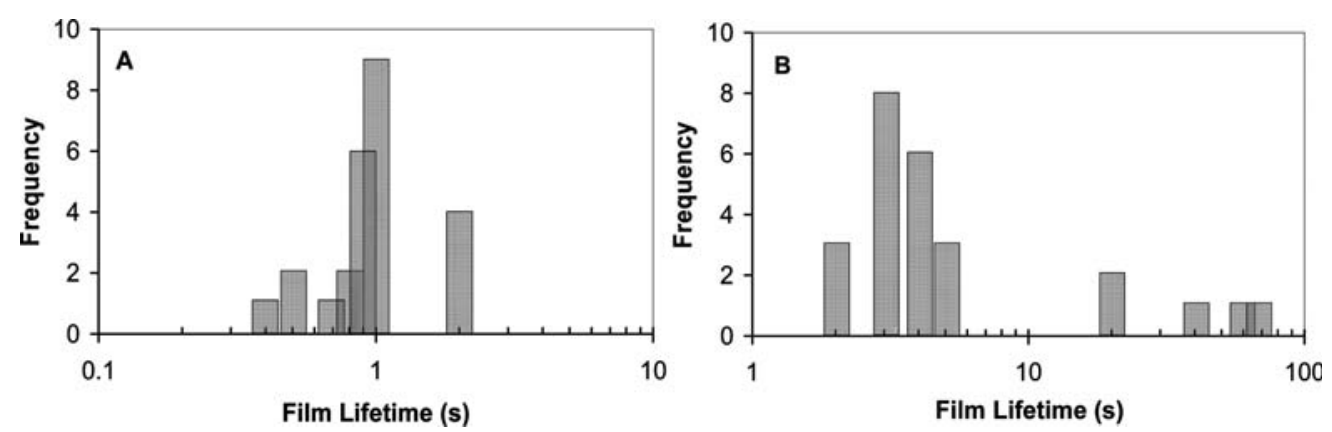

Figure 5. Variation in film lifetime in two partially inhibiting electrolyte solutions: (A) $0.06 \mathrm{M}$ NaSCN in propylene carbonate (25 films) and (B) $0.11 \mathrm{M} \mathrm{NaSCN}$ in propylene carbonate (25 films). Note the difference in time scales between (A) and (B). The minimum film lifetime, average film lifetime, and maximum film lifetime all increase at the higher concentration.

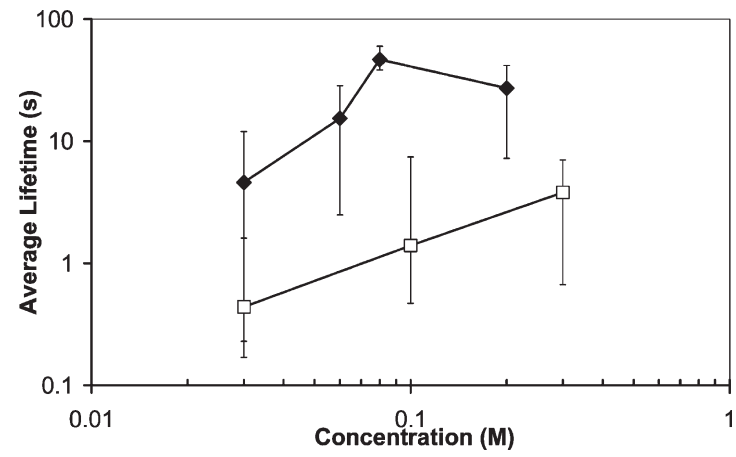

Figure 6. Average lifetime of thin films in formamide as a function of concentration in inhibiting electrolyte $\mathrm{LiCl}(\bullet)$ and noninhibiting electrolyte $\mathrm{CH}_{3} \mathrm{COONa}(\square)$ as a $\log -\log$ plot. Lifetime is higher in inhibiting than in noninhibiting salt. Lifetime error bars show the range between 10th and 90th percentiles; $13 \leq N$ $\leq 25$. Film radius is varied among and within solutions. Films formed over $20 \mathrm{~min}$ after the introduction of fresh solution become very long-lived in all cases and are not included in these averages.

concentrations than in the noninhibiting sodium acetate. This result supports a difference between films in inhibiting and noninhibiting salts. However, there is an increase in film lifetime with increasing sodium acetate concentration, despite the fact that no difference in bubble coalescence was observed in the bubble column over the concentration range used here of $0-0.3 \mathrm{M}{ }^{7} \mathrm{We}$ note that at even the highest concentration of sodium acetate studied the film lifetime was less than that measured for the lowest concentration of lithium chloride. In formamide solutions, it was observed that around 20 min after a droplet had been taken into the film holder, the films formed increased in stability and lifetime. This was observed in all solutions and in neat formamide. It is possible that the increasing stability with droplet age is due to contamination of the solution, to absorption of water from the atmosphere, or to the dissociation products of formamide. ${ }^{17}$ The droplet-age dependence of the results means that the majority of the analysis has been performed on propylene carbonate systems rather than the formamide thin films.

Thin Film Drainage and Rupture. Experimental film thickness data were obtained for suitable small, plane parallel films in a range of solutions. A single film thickness measurement in the last collected image before film rupture tells us the rupture thickness of the film. By measuring film thickness for a single film as a function of time across several images, drainage kinetics can be

(17) Porras, S. P.; Kenndler, E. Electrophoresis 2004, 25, 2946-2958.

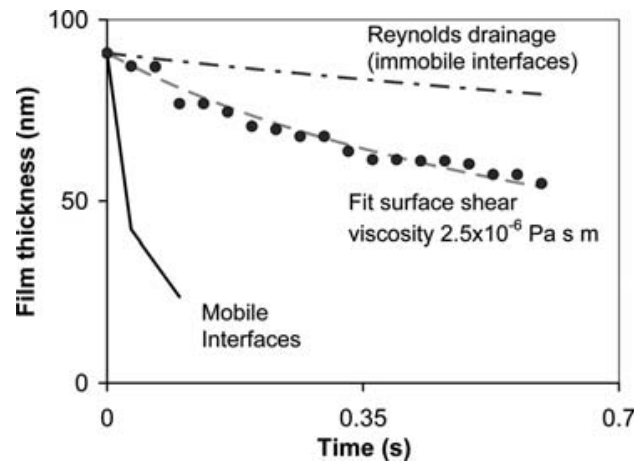

Figure 7. Comparison of experimental film drainage data $(\bullet)$ with theoretical drainage in Reynolds (- - ) and mobile interface (-) cases. Experiment data are fit with a surface viscosity of $2.5 \times 10^{-6}$ $\mathrm{Pa} \mathrm{s} \mathrm{m}(---)$. The film is in $0.15 \mathrm{M} \mathrm{LiBr}$ in propylene carbonate. Film rupture occurs at a thickness of $55 \mathrm{~nm}$.

determined and compared with existing theories (which were developed for surfactant systems). The details of the drainage rate analysis are given in the Appendix below. Such analysis was performed on one or two plane parallel films for each concentration seen here. For some electrolyte concentrations, no suitable films were obtained, either because the films produced were too large and nonplanar for all drainage rates tested or because the films were too unstable and no interference fringes were recorded for analysis before film rupture occurred. In some cases, film radius changed during drainage. This cannot be fit using existing models that assume a constant film radius; therefore, these data were not analyzed.

The fitting parameter used is the surface (shear plus dilational) viscosity, $\eta_{\mathrm{s}}$. An increase in the value of surface viscosity indicates a decrease in film drainage rate. It is emphasized, however, that we do not necessarily ascribe the observed drainage velocity changes purely to surface viscosity alteration; it is simply a convenient means to compare drainage rates between solutions, at varying film thickness and radius.

The experimental drainage rate is fit with a surface viscosity value. It can also be compared to the film thinning profile predicted by the Stefan-Reynolds equation (eq 2) for immobile interfaces. This theory is expected to provide a "lower bound" on drainage velocity. By setting the surface viscosity to a low value $\left(6 \times 10^{-8} \mathrm{~Pa} \mathrm{~s} \mathrm{~m}\right)$ in drainage analysis using eq 4 , the "upper bound" drainage velocity for fully mobile planar interfaces can also be predicted and compared with our results. An example of the comparison between fit experimental data and the two limiting cases (mobile interfaces and Reynolds) is given in Figure 7 for 
Table 1. Rupture Thickness and Fit Surface Viscosity in Plane Parallel Films

\begin{tabular}{|c|c|c|c|c|c|c|}
\hline solvent & electrolyte & $\begin{array}{c}\text { concentration } \\
\text { (M) }\end{array}$ & $\begin{array}{l}\text { lifetime }(\mathrm{s}) \pm \\
\quad 0.033 \mathrm{~s}^{a}\end{array}$ & $\begin{array}{c}\text { rupture radius } \\
(\mu \mathrm{m}) \pm 1 \mu \mathrm{m}\end{array}$ & $\begin{array}{l}\text { rupture thickness } \\
\quad(\mathrm{nm}) \pm 2 \mathrm{~nm}\end{array}$ & $\begin{array}{c}\text { surface viscosity } \\
\left(\times 10^{-6} \mathrm{~Pa} \mathrm{~s} \mathrm{~m}\right) \pm 25 \%\end{array}$ \\
\hline \multirow[t]{3}{*}{ propylene carbonate } & $\mathrm{HCl}$ & 0.14 & 0.066 & 20 & 102 & 0.34 \\
\hline & & 0.3 & 0.1 & 23 & 137 & $800^{b}$ \\
\hline & & 0.3 & 0.067 & 20 & 97 & 0.35 \\
\hline \multirow[t]{3}{*}{ propylene carbonate } & $\mathrm{NaSCN}$ & 0.06 & 0.4 & 28 & 30 & 0.49 \\
\hline & & 0.09 & 0.67 & 20 & 24 & 1.1 \\
\hline & & 0.2 & 1.36 & 17 & 34 & 5.1 \\
\hline \multirow{3}{*}{ propylene carbonate } & $\mathrm{LiBr}$ & 0.06 & 0.198 & 38 & 38 & 0.23 \\
\hline & & 0.15 & 0.594 & 20 & 54 & 2.5 \\
\hline & & 0.3 & 7 & 34 & 34 & 14 \\
\hline \multirow[t]{3}{*}{ formamide } & $\mathrm{CH}_{3} \mathrm{COONa}$ & 0.03 & 0.132 & 31 & 46.5 & 0.28 \\
\hline & & 0.1 & 0.396 & 35 & 28 & 0.40 \\
\hline & & 0.3 & 0.066 & 28 & 31.9 & 0.14 \\
\hline formamide & $\mathrm{LiCl}$ & 0.03 & 2.36 & 19 & 36.4 & 6.3 \\
\hline formamide & Nil & $0.0\left(0.02^{c}\right)$ & 0.066 & 20 & 47.8 & 0.39 \\
\hline
\end{tabular}

${ }^{a}$ The camera frame rate was 30 frames per second. ${ }^{b}$ Believed to be an atypically draining film. ${ }^{c}$ Ionic strength of dissociation products ammonium and formate in formamide. ${ }^{17}$

a typical plane parallel film, in this case in a system with partial coalescence inhibition, $0.15 \mathrm{M} \mathrm{LiBr}$ in propylene carbonate.

In this example and in all solutions for which measurement was done, the film drainage fell between the two theoretical limits; that is, drainage is more rapid than predicted by Reynolds theory for planar immobile surfaces but less rapid than plug flow between fully mobile low-viscosity interfaces. One film in $0.30 \mathrm{M} \mathrm{HCl}$ in propylene carbonate followed Reynolds drainage before rupturing at $137 \mathrm{~nm}$. However, a second film in this solution as well as a film in $0.14 \mathrm{M} \mathrm{HCl}$ in propylene carbonate showed more rapid drainage equivalent to partially mobile interfaces. The aberrant film ruptured at a very high thickness and was very unstable, and this result should be treated with caution. The surface viscosities, as well as rupture thicknesses, are presented in Table 1 for all files analyzed.

In Figure 8, the fitted surface viscosity, which increases with slower film drainage, is plotted as a function of electrolyte concentration in propylene carbonate solutions. There is a correlation between increasing concentration of inhibiting electrolyte and decrease in drainage rate (as reflected in higher surface viscosity fit). The increase in surface viscosity, which is determined from only the smaller plane parallel films, matches closely with increasing film lifetime (Figure 4 above), which is determined from all the films, including films that did not thin evenly. This is not a trivial result, because it links analysis of a particular, easily modeled case (plane parallel interfaces) with the general case of thin film stability at any film size and in the presence of surface corrugations. Further, the results correlate with the data obtained in bubble column measurements. The correlation between surface viscosity and bubble coalescence inhibition is strong evidence for the argument that electrolytes inhibit coalescence relative to pure solvent (or noninhibiting electrolytes) in part by a retardation of film drainage. This is not necessarily by a change to the surface viscosity; there is no direct information about the mechanism by which electrolytes slow drainage. Our findings indicate that deeper probing of the thin film drainage process at a molecular level may yield much useful data. For the noninhibiting electrolyte sodium acetate in formamide, the surface viscosity does not show a monotonic trend with concentration. The data have not been presented, as we are unsure that the formamide solutions used are sufficiently pure for this analysis to be meaningful.

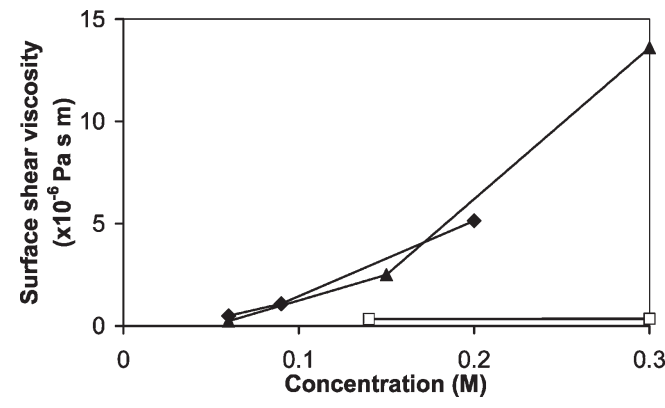

Figure 8. Fitted value of surface viscosity as a function of electrolyte concentration in propylene carbonate, for plane parallel films in solutions of inhibiting electrolytes $\operatorname{NaSCN}(\bullet)$ and $\operatorname{LiBr}(\boldsymbol{\Delta})$ and noninhibiting electrolyte $\mathrm{HCl}(\square)$. The surface viscosity parameter is used as a measure of film drainage rate. A higher surface viscosity value indicates slower film drainage. There is a strong correlation between bubble coalescence inhibition and drainage retardation.

A recent publication by Safouane and Langevin measured the surface viscosity of aqueous electrolyte solutions, during electrocapillary wave excitation. ${ }^{18}$ It was found that there was no significant viscoelasticity below $2 \mathrm{M}$ electrolyte. However, this work did not examine the concentration range over which coalescence inhibition occurs $(0.01-0.3 \mathrm{M})$, instead looking at $1.0 \mathrm{M}$ and above. The capillary waves used had a wavelength of $>1 \mathrm{~mm}$, and so the applicability to the surface relaxation of the thin films studied here (which have a diameter of $\leq 80 \mu \mathrm{m}$ ) is uncertain. Little work has been done on the viscoelastic properties of surfactant-free electrolyte solutions, and for this reason we are cautious and choose to treat the surface viscosity as primarily a fitting parameter that describes film drainage rate, without commenting on the mechanism.

Figure 9 shows film rupture thickness in propylene carbonate solutions. The film drained to smaller thicknesses before rupturing in inhibiting electrolytes $\mathrm{NaSCN}$ and $\mathrm{LiBr}$, in which rupture took place at film thicknesses between 23 and $53 \mathrm{~nm}$, than in noninhibiting electrolyte $\mathrm{HCl}$, in which films ruptured at around $100 \mathrm{~nm}$. No film was recorded in pure solvent, which is in itself an indication that rupture thickness is large. If the noninhibiting case

(18) Safouane, M.; Langevin, D. ChemPhysChem 2009, 10, 222-225. 


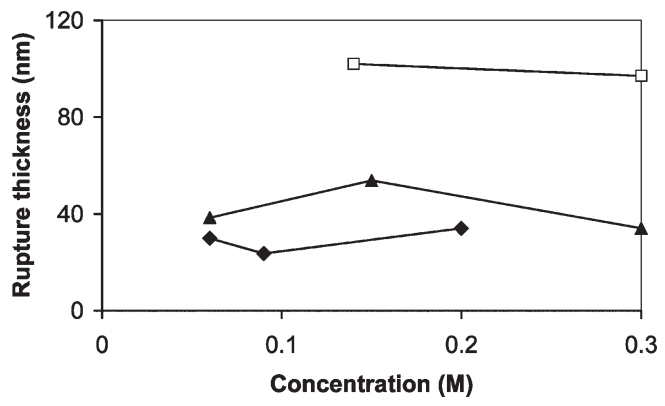

Figure 9. Film rupture thickness as a function of electrolyte concentration in propylene carbonate, for plane parallel films in solutions of inhibiting electrolytes $\operatorname{NaSCN}(\bullet)$ and $\operatorname{LiBr}(\boldsymbol{\Delta})$ and noninhibiting electrolyte $\mathrm{HCl}(\square)$. Rupture thickness is reduced in inhibiting electrolytes.

is taken as comparable, then we can conclude that electrolytes inhibit coalescence in part by allowing the thin film to drain to thinner bubble separations without rupture and coalescence occurring, in addition to retarding film drainage. There is no correlation between rupture thickness and concentration of inhibiting electrolyte in the propylene carbonate data (Figure 9). This lack of correlation is consistent with the idea that rupture is a stochastic process that can occur with increasing probability once the film drains to below a certain thickness. ${ }^{14}$

We considered the possibility that ion pairing might occur in nonaqueous solvents, particularly in propylene carbonate because of its low dielectric constant relative to water $\left(66.14\right.$ at $20^{\circ} \mathrm{C}$ compared with 80.20 for $\mathrm{H}_{2} \mathrm{O}^{19}$ ). The effect of ion pairing on bubble coalescence is in any case not conclusively known. For instance, acetic acid is known to cause foaming in aqueous systems and it is only partially dissociated, but magnesium acetate shows some ion pair formation at $0.5 \mathrm{M}$ but does not inhibit bubble coalescence in water. ${ }^{20}$ Although no study could be found in the literature on the electrolytes used here, their bubble column results reported previously ${ }^{7}$ are comparable with other electrolytes in which no evidence of ion pairing is found (alkali metal perchlorates and halides). ${ }^{21}$ At concentrations below $0.3 \mathrm{M}$, we therefore believe that ion pair formation is not a significant contributor to thin film stability.

It is not possible to draw from the thin film analysis alone conclusions about the molecular-scale mechanism by which electrolytes inhibit coalescence. A retardation of film drainage may be caused by dynamic effects such as Marangoni stresses at the air-water surface or changes in interfacial viscosity. ${ }^{12}$ It may also arise from a change in the static surface forces involving either an increase in surface repulsion or a decrease in surface attraction. ${ }^{22,23}$ A closer analysis of the drainage kinetics with more accurate initial parameters (such as surface tension gradient and Hamaker constant for the electrolyte solutions) may enable us to eliminate one or more of these possible drainage retardation mechanisms. Similarly, a decrease in the film rupture thickness might be caused by hydrodynamics (damping of interfacial deformations, for example) or by a net decrease in any repulsive surface force, enabling the surfaces to approach more closely. However, the similarity of results between water and nonaqueous solvents, in this work and in earlier bubble column experiments,

(19) Handbook of Chemistry and Physics, 80th ed.; CRC Press:Boca Raton, FL, 1999-2000

(20) Minofar, B.; Vácha, R.; Wahab, A.; Mahiuddin, S.; Kunz, W.; Jungwirth, P. J. Phys. Chem. B 2006, 110, 15939-15944.

(21) Jansen, M. L.; Yeager, H. L. J. Phys. Chem. 1973, 77(26), 3089-3092.

(22) Bergeron, V. J. Phys.: Condens. Matter 1999, 11, R215-R238.

(23) Wang, L.; Yoon, R. H. Colloids Surf., A 2005, 263, 267-274. is consistent with a mechanism of thin film stability that works on the dynamic film drainage process between gas interfaces. This is because solvent structure and equilibrium surface forces will differ widely between solvents, as the dielectric constant will be different.

\section{Conclusions}

We have shown that, in thin film drainage experiments, increased inhibiting electrolyte concentration stabilizes thin films between gas interfaces. The results are largely consistent with bulk bubble coalescence measured in bubble column experiments for propylene carbonate and formamide electrolyte solutions. In both formamide and propylene carbonate solutions, the average lifetime of thin films increases with increasing concentration of inhibiting electrolyte. In propylene carbonate, films are highly unstable in concentrations of the noninhibiting electrolyte $\mathrm{HCl}$, up to the maximum tested concentration $(0.30 \mathrm{M})$. In formamide, an increase in film lifetime in a noninhibiting electrolyte is measured, but this system may be prone to contamination of the thin films over time. Inhibiting electrolytes act both to reduce thin film drainage rate and to reduce the rupture thickness, relative to pure solvent. Both of these effects are expected to stabilize bubbles against coalescence over the lifetime of a collision. The film drainage velocity in plane parallel films lies between the two theoretical bounds for immobile, planar interfaces (Reynolds drainage) and for low-viscosity surfaces.

Acknowledgment. This collaboration was supported by travel funding from the ARC Nanotechnology Network. We acknowledge the Australian Research Council for financial support through Discovery grants (A.V.N. and V.S.J.C.) and the School of Engineering at the University of Queensland for a Fee Waiver Postgraduate Scholarship (P.T.N).

\section{Appendix: Theory of Thin Film Drainage}

The classical drainage theory describes the drainage velocity between immobile planar interfaces, which for thin films is given by the Stefan-Reynolds equation: ${ }^{24,25}$

$$
V_{\mathrm{Re}}=\frac{2 h^{3}}{3 \eta R^{2}}\left(P_{\sigma}-\Pi\right)
$$

Here, $V_{\operatorname{Re}}$ is the velocity from Stefan-Reynolds theory, $h$ is the film thickness, $\eta$ is the solution viscosity, $R$ is the film radius, $P_{\sigma}$ is the capillary pressure, and $\Pi$ is the total of the disjoining pressure including van der Waals, electrostatic, and non-DLVO components. $^{26}$

In the Stefan-Reynolds theory, the nonslip boundary condition applied at the immobile film surfaces implies that both velocity components normal and parallel to the film surfaces are zero. If the nonslip boundary condition for the parallel surface velocity component is relaxed to allow for partial mobility of the air-solution interfaces, drainage velocity will exceed that predicted by the Stefan-Reynolds theory: 8,26

$$
V=\frac{2 h^{3}}{3 \eta R^{2}} \frac{\left(P_{\sigma}-\Pi\right)}{f}
$$

(24) Reynolds, O. Philos. Trans. R. Soc. London 1886, 177, 157-234.

(25) Nguyen, A. V. J. Colloid Interface Sci. 2000, 231, 195.

(26) Karakashev, S. I.; Nguyen, A. V. Colloids Surf., A 2007, 293, 229-240. 
where $f$ is a factor which accounts for the film surface mobility and can be obtained by applying the stress boundary condition at the film surfaces, giving

$$
f=1-32 \sum_{k=1}^{\infty} \frac{1 / \lambda_{k}{ }^{4}}{6+B o \lambda_{k}{ }^{2}(h / R)+2 M a}
$$

In eq A3, $\lambda_{k}$ is the $k$ th root of the Bessel function of the first kind and zero order, $B o=\eta_{\mathrm{s}} /(\eta R)$ is the Boussinesq number which is a function of surface (shear plus dilational) viscosity, $\eta_{\mathrm{s}}$, of the film surfaces, and $M a$ is the Marangoni number which accounts for the effect of Marangoni stress on the stress balance at the film surfaces and is a function of the Gibbs elasticity. ${ }^{8,26}$

Important assumptions are made to simplify the calculations in the case of electrolyte solutions considered here.

We make the assumption that $\Pi \ll P_{\mathrm{c}}$. That is, the capillary pressure is the dominant force acting on the films at the observed rupture thicknesses $(\geq 23 \mathrm{~nm})$. The electrostatic component of the disjoining pressure can be ignored as a factor controlling drainage at these film thicknesses and high salt concentrations. The dielectric constants are 66.14 and 110.0 for propylene carbonate and formamide, respectively. ${ }^{19}$ Therefore for $0.01 \mathrm{M} \mathrm{1:1} \mathrm{electrolyte,}$ the Debye length can be calculated as $2.8 \mathrm{~nm}$ in propylene carbonate solutions and $3.6 \mathrm{~nm}$ in formamide solutions. All of the films here observed rupture at thicknesses $>20 \mathrm{~nm}$, at which thickness the surfaces are screened from each other for the range of electrolyte concentrations used. In addition, the van der Waals attraction between gas interfaces at separations $>20 \mathrm{~nm}$ is significantly less than the capillary pressure in the thin film. Therefore, the DLVO components of the disjoining pressure can be neglected.

Non-DLVO components of the disjoining pressure are likewise ignored. Steric forces due to solvation are only observed at small surface separation $(\sim 5 \mathrm{~nm}),{ }^{27}$ and so will not affect films more than $20 \mathrm{~nm}$ thick. The hydrophobic force is a controversial longrange force that attracts two hydrophobic surfaces across water. Because the existence in nonaqueous systems and at fluid interfaces is unclear, it has not been included.

Thin film capillary pressure, $P_{\sigma}$, which also appears in eq 3 , is given by

$$
P_{\sigma}=\frac{2 \sigma}{R_{\mathrm{c}}}
$$

where $R_{\mathrm{c}}$ is the radius of the film holder, constant at $2 \mathrm{~mm}$, and $\sigma$ is the solution surface tension. The surface tension used was that for

(27) Israelachvili, J. N. Intermolecular and Surface Forces; Academic Press: London, 1992. the neat solvents (given in the Supporting Information). Electrolytes are expected to influence the surface tension only weakly $(<1 \%)$ at the concentrations studied. The capillary pressure is then equal to $41.92 \mathrm{~Pa}$ for propylene carbonate solutions and $57.03 \mathrm{~Pa}$ for formamide (for $R_{\mathrm{c}}=2 \mathrm{~mm}$ ).

The second assumption is that the Marangoni number is assumed to be zero. The Marangoni number, $M a$, is given by

$$
M a \equiv E R /\left(D_{\mathrm{s}} \eta\right)
$$

where $E$ is Gibbs elasticity and $D_{\mathrm{s}}$ is surface diffusivity of species. $^{26}$ The Gibbs elasticity $E$ is in turn proportional to $(\mathrm{d} \gamma / \mathrm{d} c)^{2}$, the square of the surface tension gradient, ${ }^{28}$ which will be very small in the absence of surface active species (surface tension gradient is on the order of $1 \mathrm{mN} \mathrm{m}^{-1} \mathrm{~mol}^{-1}$, as seen in aqueous solutions ${ }^{15}$ and nonaqueous systems ${ }^{29}$ ). At these values, the error associated with measurement of change in surface tension is large, and in nonaqueous systems the possibility of contamination is high. Therefore, the solution surface tension changes are not used. A moderate correlation between Gibbs elasticity and bubble coalescence inhibition (thin film stability) has previously been reported for single electrolytes in aqueous solution. ${ }^{30}$ The reported correlation between surface tension gradient and bubble coalescence inhibition in electrolyte solutions has been further investigated by two of us using aqueous mixtures of electrolytes. ${ }^{15}$ In this study, it was clearly shown that there is no correlation between surface tension gradient and bubble coalescence inhibition for mixed electrolytes. This indicates that thin film stabilization by electrolytes is due to an influence other than Gibbs elasticity or Marangoni effects.

Given these conditions, eqs A2 and A 3 can be used to compare film drainage velocity with theoretical predictions of drainage in the presence of a fully mobile or an immobile, interface. In analysis using the Karakashev-Nguyen model, we employed the surface (shear plus dilational) viscosity, $\eta_{\mathrm{s}}$, as the relevant fitting parameter. $\eta_{\mathrm{s}}$ enters into calculation of the surface mobility factor $f$ as part of the Boussinesq number Bo. An increase in the value of surface viscosity indicates a decrease in film drainage rate. It is emphasized, however, that we do not necessarily ascribe the observed drainage velocity changes purely to surface viscosity alteration; it is simply a convenient means to compare drainage rates between solutions, at varying film thickness and radius.

Supporting Information Available: Table detailing relevant solvent properties. This material is available free of charge via the Internet at http://pubs.acs.org.

(28) Christenson, H. K.; Yaminsky, V. V. J. Phys. Chem. 1995, 99, 10420.

(29) Liu, G.; Hou, Y.; Zhang, G.; Craig, V. S. J. Langmuir, submitted for publication; doi:10.1021/la901100h

(30) Weissenborn, P. K.; Pugh, R. J. J. Colloid Interface Sci. 1996, 184, 550-563. 